\title{
Real Time Operations of the Cerebellar Cortex
}

\author{
James R. Bloedel, Vlastislav Bracha and Paul S. Larson
}

\begin{abstract}
This manuscript reviews a series of experiments which support the notion that the cerebellum and more specifically the cerebellar cortex is principally involved in real time operations required for the regulation of coordinated motor activity. Experiments are reviewed which illustrate: (1) that the climbing fiber inputs to Purkinje cells can induce a short-lasting enhancement of their responses to mossy fiber-granule cell-parallel fiber inputs, (2) that the cerebellum is not essential for the acquisition and performance of the classically conditioned nictitating membrane reflex (NMR) of the rabbit, and (3) that the observations resulting from the microinjection of lidocaine and multiple single unit recordings within the brainstem support the notion that cell populations in this region may participate in establishing the modifications in neuronal interactions required for the acquisition of the conditioned NMR. In addition, preliminary data are shown comparing the capacity of a normal subject and a patient with a massive ipsilateral cerebellar stroke to learn certain tracing tasks and to redraw these learned tracing movements $90^{\circ}$ to the orientation of the original image. The data support the notion that the cerebellum is essential, not for the initial learning of the tracing movement, but rather for performing the learned movement with the required rotation of the original image.
\end{abstract}

RÉSUMÉ: Operations en temps réel du cortex cérébral. Nous revoyons une série d'expériences qui appuient le concept que le cervelet et plus particulièrement le cortex cérébelleux est impliqué principalement dans les opérations en temps réel requises pour le contrôle de l'activité motrice coordonnée. Les expériences revues illustrent: 1) que les influx des fibres grimpantes aux cellules de Purkinje peuvent induire un rehaussement de courte durée de leurs réponses aux influx fibres moussues - microglie - fibres parallèles, 2) que le cervelet n'est pas essentiel à l'acquisition et au fonctionnement du réflexe conditionné de membrane nictitante (RMN) du lapin, et 3) que les observations résultant d'enregistrements multiples d'unités après micro-injection de lidocaïne à l'intérieur du tronc cérébral appuient le concept que certaines populations cellulaires dans cette région peuvent participer à l'établissement de modifications dans les interactions neuronales requises pour l'acquisition du réflexe conditionné RMN. De plus, nous présentons des données préliminaires comparant la capacité d'un sujet normal et d'un patient ayant un infarctus cérébelleux ipsilatéral massif à apprendre certains tracés et à redessiner ces tracés avec une rotation de $90^{\circ}$ par rapport à l'image originale. Ces données appuient le concept que le cervelet n'est pas essentiel à l'apprentissage initial du mouvement requis pour exécuter le dessin, mais bien à l'exécution du dessin avec une orientation différente de celle de l'image originale.

Can. J. Neurol. Sci. 1993; 20 (Suppl. 3):S7-S18

The precise operations performed by the cerebellum in modifying the activity in central pathways involved in motor control are currently unknown. At present there is even a lack of agreement on the class of functions for which the cerebellum is responsible. One popularized view is that the principal role of this structure is the acquisition and storage of the plastic changes underlying learned and/or conditioned motor behaviors. ${ }^{1-6}$ An alternate view proposes that, although the cerebellum is likely involved in skill acquisition, its most critical contribution to regulating motor behavior relates to the real time, on-line processing it performs in order to optimize the output of the motor system during the performance of coordinated, novel movements. ${ }^{7,8}$ This paper reviews the findings obtained in our laboratory supporting this view and relates these observations to hypotheses that are currently serving as a framework for our ongoing studies of cerebellar cortical function.

The argument that the cerebellum serves as a storage site critical for the engrams required for motor learning receives its strongest support from two sets of findings: (1) experiments showing that the climbing fiber input is capable of producing a long term depression of Purkinje cell responsiveness to parallel fiber inputs, ${ }^{1,2}$ and (2) studies indicating that specific cerebellar lesions and/or pharmacological manipulations of the cerebellar cortex or nuclei selectively affect conditioned responses in classical conditioning paradigms while leaving the unconditioned responses unaffected. ${ }^{3.5 .6 .9}$

Long-term depression has been observed independently in different laboratories using the conjunctive stimulation paradigm. 1,2.10,11 This effect is produced by pairing the stimuli that activate the parallel fiber system and the climbing fiber inputs to the same population of Purkinje neurons. In general this approach requires maintained activation of the climbing fiber system at rates that are comparatively high compared to those observed under more behavioral conditions. Recently, rates of climbing fiber stimulation at $1 \mathrm{~Hz}$ applied for several seconds have been effective in producing long term depression.

From the Division of Neurobiology, Barrow Neurological Institute, St. Joseph's Hospital and Medical Center, 350 West Thomas Road, Phoenix, Arizona Reprint requests to: James R. Bloedel, Division of Neurobiology, Barrow Neurological Institute, 350 West Thomas Road, Phoenix, Arizona, U.S.A. 85013 
However, using rates this low produces this effect in less than half of the cells studied. ${ }^{10}$ Furthermore, phenomena relatable to long-term depression never have been directly demonstrated using natural stimuli.

The ablation studies supporting the memory hypothesis all share a common feature. They have demonstrated that lesions of specific cerebellar output pathways selectively modify some types of conditioned behavior. However, these observations do not provide direct support for the argument that the most critical storage site for the memory traces underlying motor learning resides in this structure. ${ }^{8}$ Nor is direct support for this postulate provided by studies using electrical stimulation in various cerebellar afferent pathways as either the conditioned or the unconditioned stimuli. ${ }^{12-15}$ Furthermore, experiments involving patients with cerebellar pathology presented as support for the cerebellar learning hypothesis ${ }^{16}$ actually illustrate that these patients can improve the performance of a skilled movement with practice (See Bloedel ${ }^{7}$ for further discussion). Consequently these clinical data illustrate the involvement of the cerebellum in task acquisition and performance but do not provide evidence that this structure is a required storage site for the underlying plastic processes.

The experiments reviewed here address the type of operation performed by the cerebellum. First, data will be presented supporting the view that the activation of a climbing fiber input to a Purkinje cell produces a short-term enhancement of Purkinje cell responsiveness to parallel fibers. These observations will be related to hypotheses regarding the possible functional role of the cerebellar sagittal zones. Second, studies will be presented challenging the view that the cerebellum is a required storag $f$ site for the memory traces produced by the classical conditioning of the nictitating membrane reflex in the rabbit. In addition, related experiments will illustrate that there are alternate sites within the brainstem which are at least equally probable locations for the plastic changes underlying the establishment of this type of conditioned behavior. Third, a new series of experiments will be presented providing preliminary data regarding the nature of the integration occurring in the cerebellum.

\section{Cerebellar Cortical Operations}

\section{Methods}

\section{Passive Stimulation Paradigms}

The first series of experiments (See ${ }^{17}$ for additional description of the methods) employed cats that were decerebrated at the rostral margin of the superior colliculus and paralyzed with galamine triethiodide. The initial procedures, including the decerebration, were done while the animal was anesthetized with Halothane. Following the decerebration and the appropriate exposure of the cerebellar cortex, the anesthesia was discontinued. The animal's head was stabilized for recording studies using a standard stereotaxic head holder. Recordings were made with glass micropipettes filled with $4.0 \mathrm{~N}$ sodium chloride.

The paradigms generally employed a paw tap, joint flexion, or stimulation of a parallel fiber beam to activate the mossy fiber/parallel fiber input at specified times following the spontaneous occurrence of the same cell's climbing fiber input. Briefly, the simple and complex spikes were discriminated separately, and a pulse indicating the time of occurrence of the complex spike was used to trigger the stimulus generator after a specifiable delay. The amplitude of histograms obtained when the stimulus was not coupled to the complex spike was compared with that of the histograms constructed from trials in which the surface stimulus was triggered at a specific time following the cell's climbing fiber input. A gain-change ratio was calculated to quantify this comparison:

(Amplitude of coupled response relative to background)

(Amplitude of uncoupled response relative to background)

\section{Effects of Climbing Fiber Inputs on Purkinje Cell Activity in Ambulating, Decerebrate Ferrets and Cats}

In order to examine the effects of climbing fiber inputs on the simple spike responses of a small population of simultaneously recorded Purkinje cells in a more behavioral context, a perturbed locomotion paradigm was developed ${ }^{18}$ in which it was possible to record from up to ten Purkinje cells in identified sagittal zones. ${ }^{19}$ These experiments first were performed using ferrets. More recent experiments have employed cats. All animals were decerebrated under Halothane anesthesia just rostral to the superior colliculus at an angle appropriate for maintaining their capacity to ambulate with only the stimulation of the treadmill moving under foot. A glue cap was constructed on the skull so that the head of the animal could be stabilized for recording by attaching it loosely to the stereotaxic bar. The limb ipsilateral to the cerebellar recording site was perturbed intermittently by interjecting a rod into the trajectory of the forelimb's swing phase approximately every ninth step as the animal locomoted.

All Purkinje cell activity recorded in these experiments employed one or two arrays of five sagittally-aligned electrodes separated at the tip by approximately 180 microns. In some of these experiments the EMG activity of the biceps and triceps was recorded during the stepping behavior. All data were processed using the RTPR analysis described originally by Lou and Bloedel. ${ }^{19}$ The pertinent aspects of this method will be briefly reviewed when the specific results from these studies are presented.

\section{Results}

The initial experiments were designed to determine the changes in the responsiveness of Purkinje cells to their mossy fiber/parallel fiber input following the occurrence of a climbing fiber input activated either spontaneously ${ }^{17}$ or in response to natural tap stimuli or small flexions of the wrist joint. ${ }^{20}$ The experiment shown in Figure 1 examined the changes in responses occurring after spontaneously-occurring climbing fiber inputs. This study ${ }^{21}$ was designed to ensure that only interactions occurring within the cerebellum could be responsible for the observed changes in simple spike activity following the occurrence of the climbing fiber input.

In this experiment the parallel fibers were activated directly by a stimulus applied to the folial surface: (1) either randomly with respect to the time of occurrence of the cell's climbing fiber input, or (2) triggered at specified time intervals following the occurrence of spontaneous climbing fiber inputs to this neuron. Figure 1 illustrates the effect of the stimulus on two different cells, one with an excitatory response to the surface stimulus $(A-C)$, the other with an inhibitory response to the same 


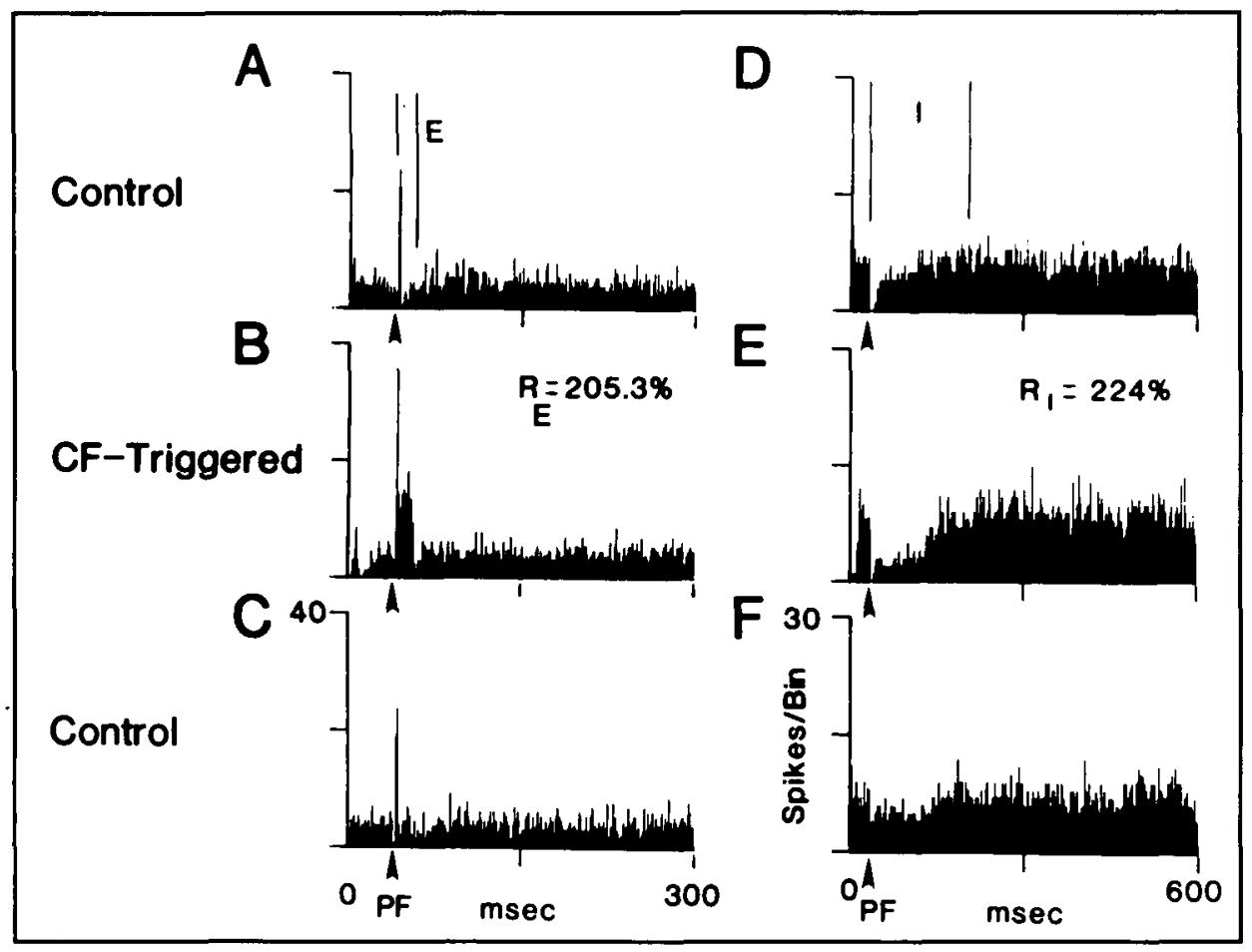

Figure I - Effect of spontaneous climbing fiber inputs on the responsiveness of Purkinje cells to parallel fiber inputs. A-C and D-F show the responses of two different Purkinje cells to a parallel fiber volley evoked by an electrode placed on the surface of the same folium. $A$ and $C$ : The surface stimulus was applied randomly relative to the cells' climbing fiber inputs. $B$ and $E$ : Responses of the same cells to the same intensity surface stimulus when applied at the indicated time after the occurrence of the cells' climbing fiber inputs. $C$ and $F$ : Responses when the same surface stimulus was again applied randomly relative to the climbing fiber inputs. The trials used to construct these histograms were obtained immediately after those used to construct the histograms in $B$ and E. Each histogram was constructed from 50 trials. From 21

condition (D-F). In A and $\mathrm{C}$ the surface stimulus was applied at times which were not coupled to the occurrence of the cell's climbing fiber input. $\mathrm{B}$ and $\mathrm{E}$ show the effects of applying the surface stimulus at approximately $35-40 \mathrm{msec}$ following the occurrence of each cell's complex spike. Notice that in both cases the simple spike response was enhanced. When the stimulus was again applied randomly relative to the cells climbing fiber input ( $\mathrm{C}$ and $\mathrm{F}$ ), the enhancement was no longer present. These trials were performed immediately after those in B and $E$, indicating that the effect of the climbing fiber input shown in $B$ and $E$ was very short lasting. Stimulus interval studies ${ }^{17}$ revealed that this effect peaked early (approximately $35-50$ $\mathrm{msec}$ ) and persisted to approximately $150 \mathrm{msec}$, although in a few cells some increased responsiveness was present for as long as $250 \mathrm{msec}$.

Observations obtained in several other related studies support the findings in Figure 1. The methods employed in the other experiments utilized a tap of the paw or a modest dorsiflexion of the wrist to activate a combined simple and complex spike response in a given Purkinje cell. ${ }^{20,22}$ All trials were then segregated into two groups. One group consisted of those in which no complex spike occurred in response to the natural stimulus, whereas the other consisted of the trials in which complex spikes were evoked. Histograms then were constructed from these two groups of trials and normalized on the basis of trial number. This procedure made it possible to compare the amplitude of simple spike responses in trials in which the climbing fiber input was evoked with the amplitude of those in which this input was not activated. As the data from Figure 1 would predict, enhanced simple spike responses were clearly present in those trials in which the climbing fiber input also was evoked. ${ }^{20}$ Again enhancements of both excitatory as well as inhibitory responses were observed.
Together these data clearly show that the simple spike responsiveness of a Purkinje cell is enhanced for a brief period following the activation of its own climbing fiber input and that this enhancement can occur for excitatory as well as inhibitory response components.

The next series of experiments performed in our laboratory were designed to determine if the short term enhancement of simple spike responsiveness was associated with the activation of climbing fiber inputs in a more behaviorally-relevant paradigm. The studies were also performed to initiate an investigation into the functional operation performed by the cerebellar sagittal zone. Using the multiple electrode array described above, the responses of up to six sagittally-aligned Purkinje cells (assuming two recorded on one electrode) could be recorded simultaneously in either one or two different zones. A data processing technique was developed to assess quantitively the simple spike responses recorded from each small population of isolated Purkinje cells on a trial-by-trial basis. This method, called the RTPR (Real Time Postsynaptic Response ${ }^{19}$ ) digitizes each of the recording spike trains, converts the occurrence of each spike to a simulated IPSP, and linearly sums these postsynaptic potentials assuming that each recorded Purkinje cell terminates, at least in part, on the same postsynaptic neuron. This analog signal can then be summed over a response window to provide a measure of population simple spike response amplitude in a single trial. The assumptions underlying the application of this method have been discussed previously ${ }^{19}$ and consequently will not be reviewed here.

Figure 2 illustrates the most significant finding from these experiments. The data in this figure (from ${ }^{19}$ ) were obtained from two separate trials in an acutely decerebrate ambulating ferret. In one trial (A-I) the animal's locomotion is unperturbed and in the other (J-R) the locomotor cycle was perturbed as described 


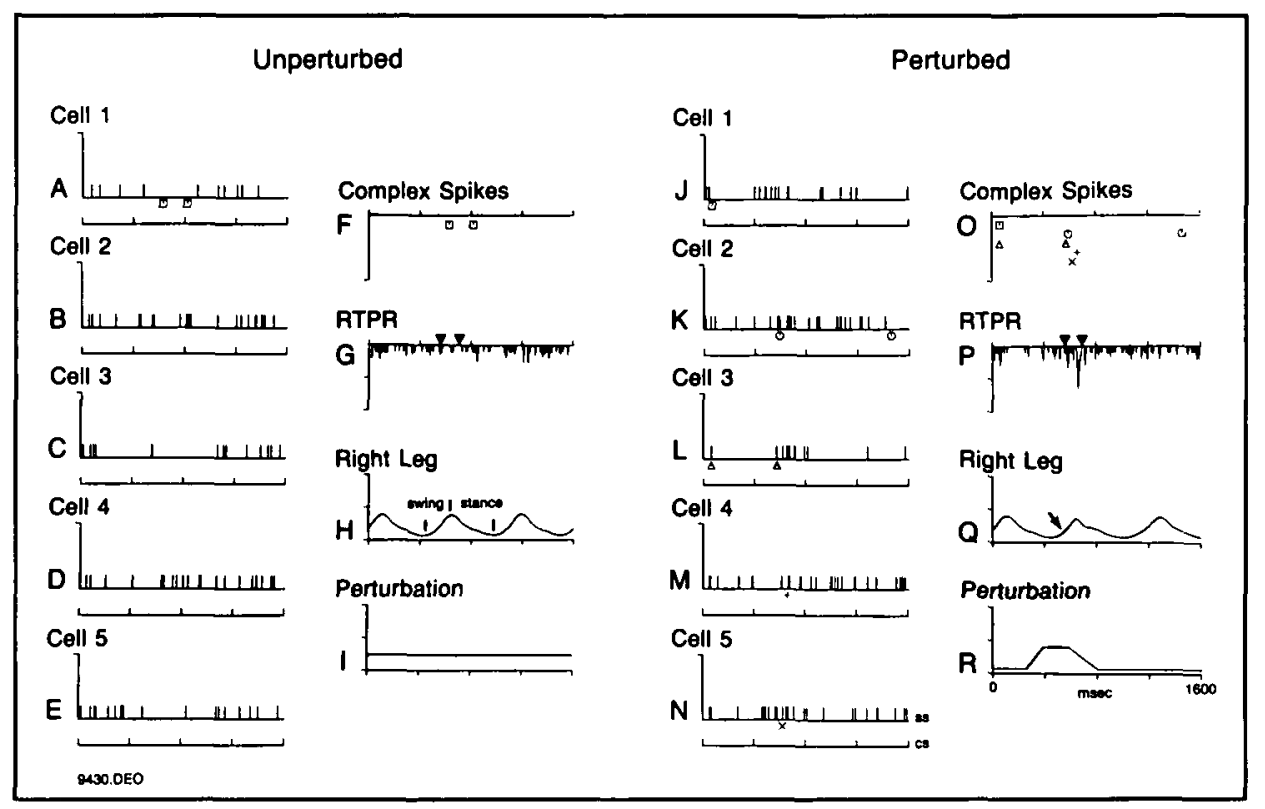

Figure 2 - Single trial analysis of simple and complex spike activity resulting from perturbation of the locomotor cycle. This figure shows the analysis of the simple and complex spike activity recorded from five cells simultaneously during an unperturbed $(A-I)$ and a perturbed $(J-R)$ trial. The digitized simple spikes for each trial are shown in A-E and J-N, respectively. The time at which the complex spikes for each cell occurred are shown beneath each time base with different symbols. The composite complex spike activity for each trial is shown in $F$ and $O$. respectively. $G$ and $P$ illustrate the RTPR calculated from the simple spikes of the simultaneously recorded newrons in each trial. The time course of the movement of the perturbation bar into the trajectory of swing phase is show'n in $R$. $H$ and $Q$ show the potentiometer output signalling the forward and backward movement of the forelimb ipsilateral to the recorded unit activity. The arrow in $Q$ designates the time at which the forelimb contacted the bar: From "'.

in Methods. In A-E and $\mathrm{J}-\mathrm{N}$ the time of occurrence of each cell's complex spike is shown underneath the corresponding digitized simple spike record. The times of occurrence for all the complex spikes in each trial are shown in $\mathrm{F}$ and $\mathrm{O}$, respectively. The simple spike responses for this set of neurons in both the unperturbed and perturbed trial were combined to generate the RTPRs shown in $G$ and $P$, respectively. The time course of the steps in each trial are shown in $\mathrm{H}$ and $\mathrm{Q}$, with the arrow in $\mathrm{Q}$ indicating when the limb hit the perturbation bar.

Notice that in the unperturbed trial the complex spike responses of the recorded neurons do not occur preferentially at a given phase during the step cycle. Furthermore there is very little if any simple spike modulation detectable in this single trial. The observations are quite different in the perturbation trial. The record showing the time of occurrence of the complex spike responses for all the cells $(O)$ clearly indicates that the complex spikes of four of the five neurons were activated shortly following the time at which the forelimb contacted the bar, the time indicated by the arrow in Q. Associated with this synchronous activation of climbing fiber inputs, there was also an enhanced modulation of the population's simple spike activity, as revealed by the difference in the RTPR between the perturbed $(P)$ and unperturbed $(G)$ trials.

The synchronous activation of climbing fiber inputs to sagittally-aligned Purkinje cells in this region and the associated enhancement of simple spike modulation was a very consistent, statistically significant finding throughout these experiments. Furthermore the extent of the modulation was statistically correlated with the proportion of Purkinje cells whose climbing fiber inputs were activated within the response window. In addition, an analysis of the data using a modification of the gain-change ratio calculation ${ }^{17}$ provided further evidence that the enhanced simple spike modulation was related to the extent to which the perturbation activated the cells' climbing fiber inputs.

\section{The Cerebellum and Motor Learning}

The initial experiments performed in our laboratory addressing the role of the cerebellum in motor learning have focused on determining whether this structure is in fact necessary for the acquisition of different types of conditioned behavior, as suggested by several studies reviewed above. Other studies presented in this section examined whether other brainstem sites may be critically involved in this process.

\section{Methods}

The experiments employed two different preparations: (1) a decerebrate, decerebellate rabbit preparation, and (2) an intact chronic rabbit instrumented for the microinjection of lidocaine into specific brainstem nuclei or the recording of multiple single unit activity in these regions. The methods related to the decerebrate, decerebellate rabbit preparation have been described extensively elsewhere. ${ }^{23}$ In brief, rabbits were decerebrated under Halothane anesthesia by placing multiple electrolytic lesions across the brainstem at a level just rostral to the superior colliculus and the red nucleus. When the protocol required removal of the cerebellum, this structure was removed by aspiration either following the acquisition of the task or prior to the commencement of any conditioning. A standard paired-trial delay conditioning paradigm was used, employing a tone as the conditioned stimulus $(500 \mathrm{~Hz})$, and in most animals an airpuff applied to the animal's cornea was used as the unconditioned stimulus. The interstimulus interval ranged from 250 to 350 $\mathrm{msec}$, and the two stimuli were co-terminated. The intertrial intervals were varied between 8-10 sec.

The experiments examining the effects of microinjections of lidocaine in brainstem nuclei used intact, awake rabbits. The methods employed were described recently by Bracha et al..$^{24}$ Under sterile surgical conditions each animal was instrumented in such a way that the apparatus required for monitoring movement of the nictitating membrane and for applying the airpuff 
stimulus could be mounted on the head firmly without requiring that the head be stabilized beyond that provided by a standard rabbit restraint box. In addition guide tubes were inserted stereotaxically over the brainstem nuclei of interest. Paired conditioned and unconditioned stimuli were applied using a typical delay paradigm with an interstimulus interval of $350 \mathrm{msec}$ and pseudorandomly distributed intertrial intervals ranging from 19 $21 \mathrm{sec}$. The conditioned stimulus was a $1000 \mathrm{~Hz}, 85 \mathrm{~dB}$ tone, and the unconditioned stimulus was a corneal airpuff. Once the animals were conditioned, microinjection of lidocaine was accomplished through small 31 gauge tubes inserted into the stereotaxically-positioned guide tubes. Only one injection site was explored each day in each animal. As indicated in Bracha et al., ${ }^{24}$ a control series of experiments was performed in which saline rather than lidocaine was injected in the same brainstem sites. To test for effects of the injection on both conditioned and unconditioned responses, paired CS-US trials were alternated with US alone trials, making it possible to assess changes in the UR without contamination due to a preceding CS and CR.

In another group of rabbits, a newly-developed multiple single unit recording system was implanted to enable the recording of up to twelve neurons in the same region receiving the injection of lidocaine in the previously described study. This recording system consisted of three bundles of four $25 \mu \mathrm{m}$ microwires inserted through guide tubes to stereotaxically-determined targets in the brainstem. Each group of four wires could be manipulated individually. The recordings were stable enough to permit the recording of the isolated units throughout an entire 2-3 hour training session. Rabbits in which the array was implanted underwent paired trial conditioning using a paradigm identical to that described above for the lidocaine injection studies. Before each daily session began, an attempt was made to isolate as many neurons as possible before commencing with the paired trials.

\section{Results}

The capability of the decerebrate, decerebellate rabbit to acquire the classically-conditioned nictitating membrane reflex is shown in Figure 3. This plot, taken from Kelly et al., ${ }^{23}$ illustrates the data from an experiment using this preparation in which the conditioned behavior could be repeatedly acquired and extinguished as long as the viability of the preparation was maintained. During conditioning, a succession of paired CS-US trials were presented until the percent CRs reached a maximum. Tone only trials then were employed to extinguish the behavior. The conditioned behavior could be reacquired when the presentation of paired trials was resumed. These findings are also consistent with other experiments in our laboratory, reported in abstract form, ${ }^{35}$ in which conditioned modifications of gait were obtained in decerebrate ferrets whose cerebellum had either been completely or partly removed. Lesioned animals modified their stepping movements until the perturbed extremity achieved a smooth trajectory during swing phase as the paw passed over the bar.

We have interpreted these data as indicating that the previously reported effects of cerebellar ablation on the conditioned nictitating reflex were not the result of ablating the site at which the engrams are stored. We have previously hypothesized that these observations are due to disrupting a critical action of cerebellar output projections on brainstem nuclei required for establishing these plastic changes. If so, it should be feasible to localize regions within the brainstem at which small lesions can produce selective effects on the conditioned response behavior.

The experiment shown in Figure 4 illustrates that such sites exist outside the cerebellum, even on the afferent-most side of the reflex circuitry. Bracha et al. ${ }^{10}$ used microinjections of lidocaine to temporarily block neural activity in the medial region of the pars oralis of the spinal trigeminal nucleus and the adjacent reticular formation. The plot in $\mathrm{A}$ shows that the injection of

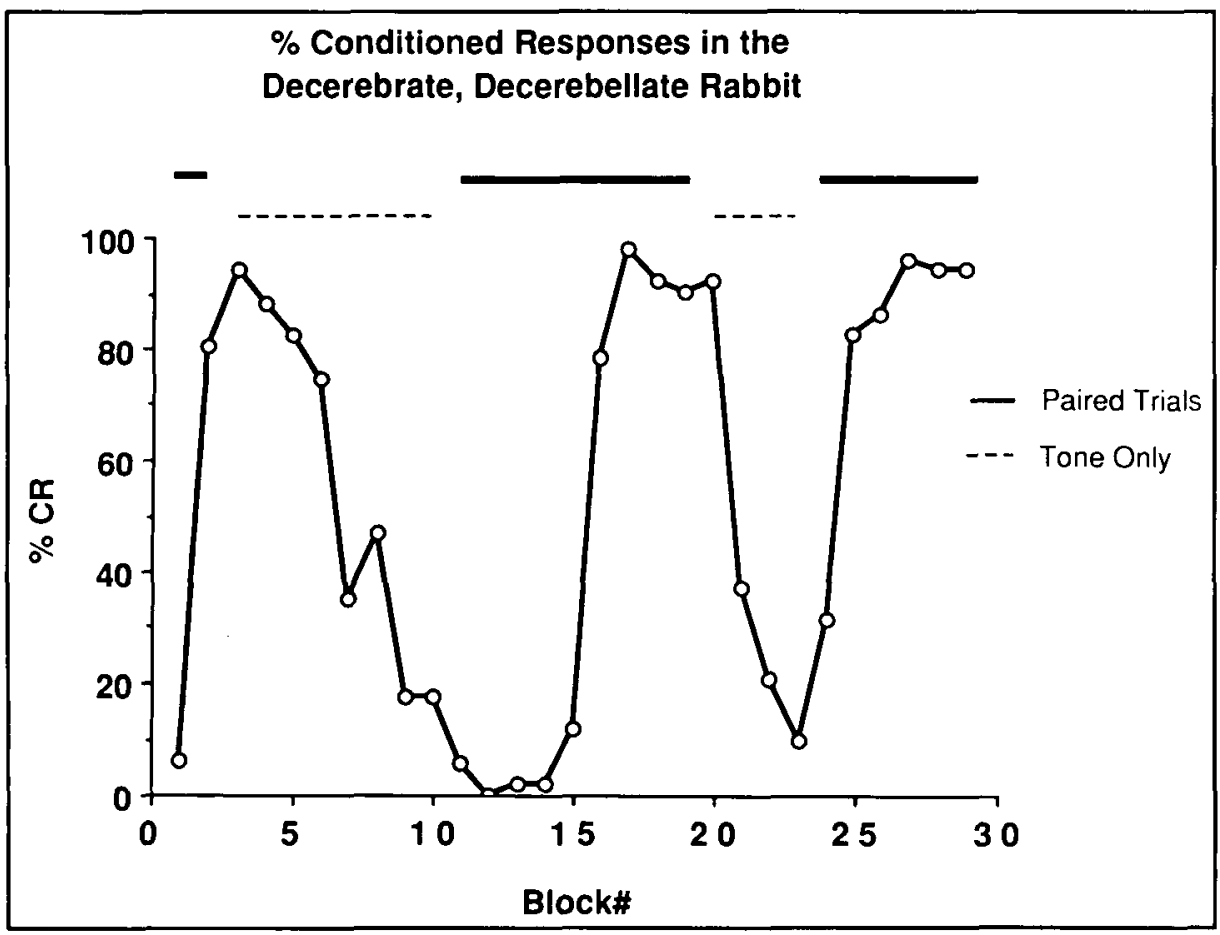

Figure 3 - Acquisition and extinction of conditioned nictitating membrane refleves in a decerebrate, decerebellate rabbit. This plot show's the changes in the percent of conditioned responding as the animal was repeatedly exposed first to blocks of 50 trials consisting of paired conditioned and unconditioned responses and then to blocks of trials in which only the conditioned stimulus (tone) in applied. From $^{2.3}$. 


\section{NMR Changes After Lidocaine Injection into the Trigeminal Nucleus}

A

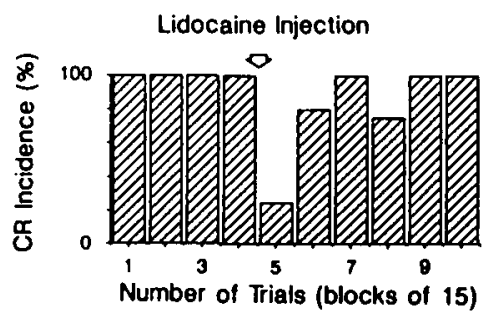

B

\section{CRs in Tone+Air Puff Trials}

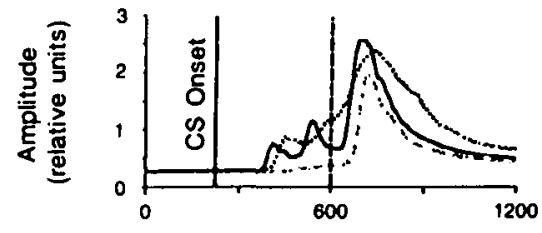

\section{C}

URs in Air Puff Only Trials

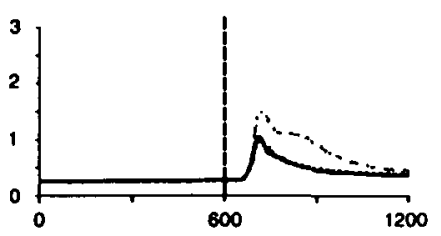

D

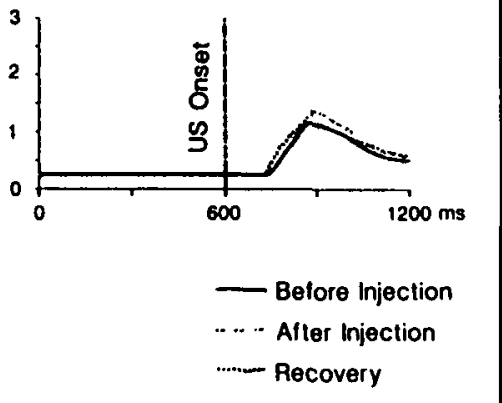

Figure 4 - Effect of lidocaine injection at the junction of the pars oralis of the spinal trigeminal nucleus and the adjacent reticular formation. $\boldsymbol{A}$ : The effect of the lidocaine injection on incidence of the conditioned response. Each bar indicates the average percent responding over a block of 15 trials. B: An overlay of the nictitating membrane response in three different trials, one before. one after, and one at the peak effect of lidocaine injection. $C$ : The unconditioned nictitating membrane responses in three airpuff-only trials during the control period, at the maximum effect of the lidocaine injection, and after recovery from the injection. D: The unconditioned responses evoked by a light unconditioned stimulus at the same three periods during the experiment as in $B$ and $C$. This control was performed to ensure that the change in the responses occurring following lidocaine injection were not due to its direct action on the output pathways responsible for evoking the reflex. From ${ }^{24}$. lidocaine produced a substantial reduction in the incidence of conditioned responses for a short period. The characteristics of the conditioned and unconditioned responses before, during and after the maximal effects of lidocaine are apparent in the three superimposed trials in $B$. Note the dramatic reduction in the amplitude of the conditioned response resulting from the injection.

The effects of the injection on the unconditioned response was assessed on airpuff-only trials that were interdigitated with the paired stimulus trials. In the animals reported in this study either the lidocaine injection had no effect when localized to the region of the medial pars oralis or it produced a slight increase in the amplitude of this response, as shown in $\mathrm{C}$. To show that the reduction in CR amplitude was not due to a direct effect of the lidocaine on the output of the motor nuclei, the consequence of the injection on the UR to a light stimulus was determined (D). The lidocaine injection clearly did not reduce this response. These data clearly elucidate one brainstem site outside the cerebellum at which temporary blockade of neural activity can produce selective depression of the conditioned response. A similar selective effect on the $\mathrm{CR}$ was observed following lesions at a more rostral brainstem site referred to as the supratrigeminal region. ${ }^{26}$

To further examine the contribution of this brainstem region to the conditioning of the nictitating membrane reflex, a technique was developed to examine the changes in the response characteristics of up to twelve simultaneously recorded neurons over the entire time required for the acquisition of the conditioned behavior. ${ }^{27}$ Figure 5 shows an example of the recordings obtained from four neurons simultaneously during two trials in which paired CS-US were applied. In one trial only a UR was evoked (A); in the other (B) both a CR and UR were observed. In both trials the unitary activity was highly correlated with any movement of the nictitating membrane associated with either the CR or UR.

Our initial findings from these experiments indicate that unitary activity in the region of the pars oralis of the spinal trigeminal nucleus undergoes modifications in response properties that parallel the acquisition of the conditioned behavior. In somewhat over half the cells the responses were highly correlated with characteristics of the conditioned or unconditioned responses. Other neurons underwent changes in their response properties which were related to the time course of the conditioning process rather than the changes in the amplitude or incidence of the conditioned and unconditioned responses. Together with the injection studies, these findings indicate that this brainstem region may be one of the putative sites for the plasticity required for the conditioning of this reflex behavior.

\section{Motor Learning and Motor Performance in a Cerebellar Patient}

The studies in the previous two sections strongly suggest that the circuitry of the cerebellar cortex is well suited for a role in the on-line operations required to optimize the coordination of motor behavior and that the cerebellum is not required for the acquisition of at least certain types of motor learning. Our laboratory also is interested in the nature of the integration the cerebellum performs through the implementation of these operations during the execution of voluntary movements. A recent review ${ }^{7}$ proposed that the cerebellum integrates visuomotor information 


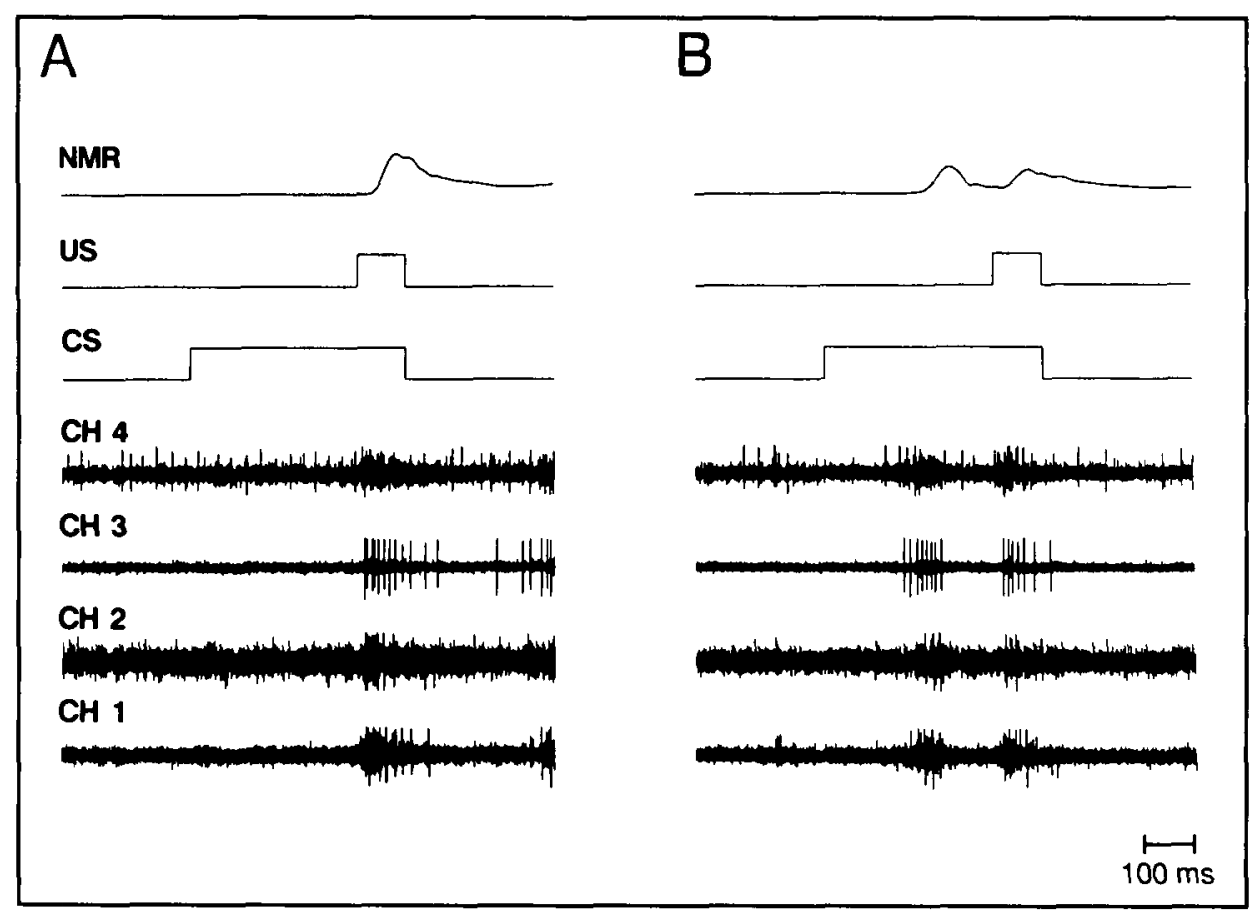

Figure 5-Illustration of the multiple single unit recording during the acquisition of conditioned responses in a previously naive rabbit. Two different trials in which paired CS - US stimuli were applied are shown. A: Trial in which only a $U R$ was evoked. B: Trial in which both the $C R$ and $U R$ were evoked during the same acquisition period. The data on channels $I-4$ were acquired with the microwire recording system described in the text. CS: Conditioned stimulus. US: Unconditioned stimulus. NMR: Mechanogram of the nictitating membrane movement.

with other information reflecting peripheral inputs, activity in descending pathways and inputs reflecting body scheme and an intrinsic representation of target space. It also was argued that this type of multisensory integration cannot be performed by other central structures. In this section the initial findings from a new series of experiments attempting to examine the characteristics of this integration are reported. The experiments compare the data from cerebellar patients and normal subjects in a series of two dimensional tracing tasks in which it is necessary to generate modifications in either the outline or orientation of the figure in order to perform the task successfully.

\section{Methods}

The experiments were designed to study aspects of motor learning as well as to assess the subject's capacity to perform on-line calculations required to modify the relationship of the traced image relative to an original template and to transpose it in different spatial orientations. The subject was a patient with an MRI-documented lesion restricted to but including the lateral and intermediate cerebellum. The control subject is age and sex matched to the patient.

In each of the tasks described below the subjects were given no restrictions regarding their performance except that each tracing must be completed within a six second period. Each task was performed over a block of 20 consecutive trials, and each was initiated at a time chosen by the subject. In these initial experiments error of performance was assessed only in the spatial domain. This was determined by calculating the area between the desired tracing path and the actual path traced by the subject. In those trials in which an image was drawn without an underlying template, an "ideal" outline based on the desired trajectory was placed over the outline of the subject, the best fit of the two outlines was determined, and the error was calculated.

\section{Results}

In the first task the subjects were asked to trace the irregularly shaped outline shown in Figures 6 and 7 over twenty consecutive trials. The age-matched normal subject (Figure 6) clearly had very little difficulty with this task. His performance was very accurate even on the first trial and improved to a maximal level of performance (minimal error) over the first few trials. The cerebellar patient also was able to improve his performance as the tracing movement was practiced. As shown in Figure 7, the patient's performance improved from trial 1 to trial 3 as judged by the decreased difference between the initial template and the drawn image.

Next the subject was asked to redraw the object with its axis rotated 90 degrees and without any underlying template as a guide. After each trial the rotated template was placed over the drawn image to permit the subject to assess its actual shape relative to that of the template. The age-matched control subject was able to perform this task very well (Figure 6). Notice that after 19 trials the subject was clearly able to draw a figure which very closely resembled the original in both size and form. In fact, even in the first trial the drawing qualitatively resembled the template. In contrast the cerebellar patient had considerable difficulty in making the necessary transposition to draw the figure accurately, independent of the number of trials performed (Figure 7). Control experiments substantiated that the deficit in the patient's performance was greater when a rotation of the object was required than when it was not. The cerebellar patient also performed poorer than the control in tasks requiring that the object be traced using a line that was equidistant from the template around its entire circumference.

Although these data need to be substantiated by a larger subject population, the data clearly indicate a deficit in the cerebellar patient that is accentuated when complex visuomotor 


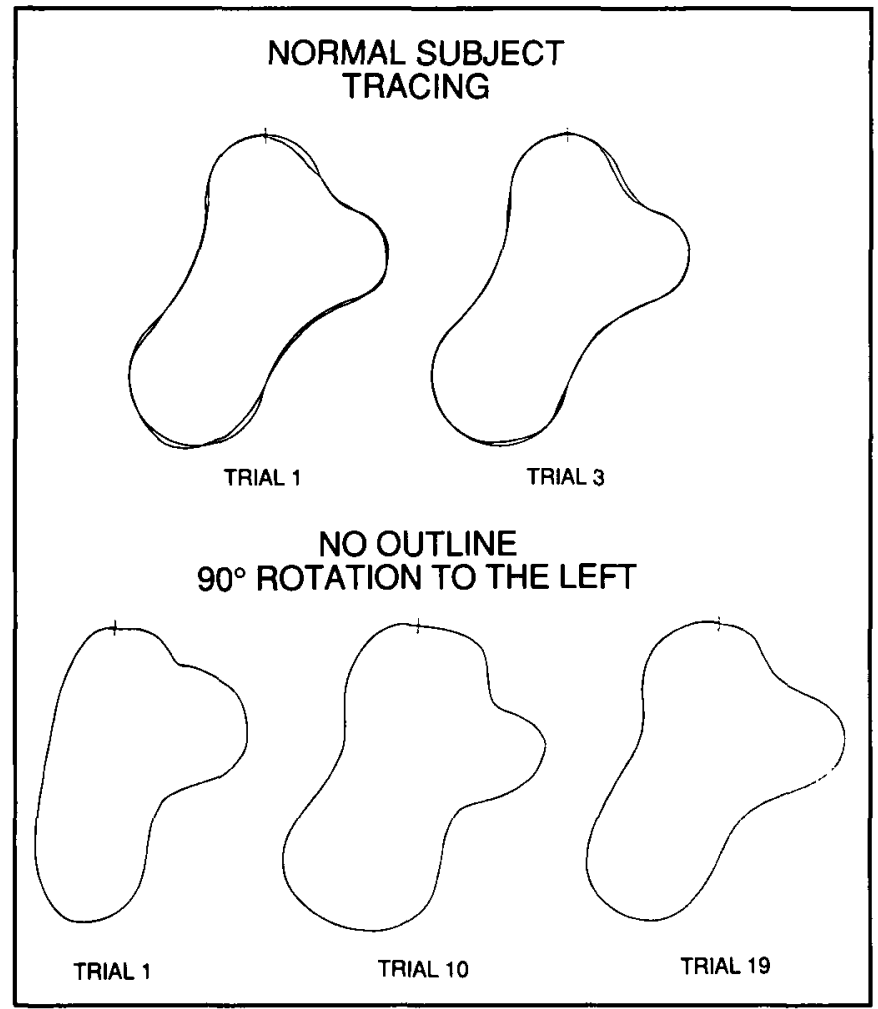

Figure 6- Ability of a normal subject to draw an irregularly shaped object under iwo different conditions. In the experiment shown in the top half of the figure. the subject was asked to trace an irregularly shaped object as accurately as possible within 6 seconds. In the experiment shown in the lower half of the figure, the subject was asked to draw the same figure rotated 90 degrees to the left and without a template. However between trials the template was placed over the drawing so that the accuracy of the performance could be assessed as the experiment progressed.

processing is required. Note also that the cerebellar patient was able to improve his performance when this type of complex integration was not required, namely when the task consisted only of tracing the outline of a template that was continuously visible and directly underneath the outline being drawn by the subject. The implications of these findings will be discussed in greater detail below.

\section{General Overview}

\section{Cerebellar Cortical Operations}

In our view the above observations support the argument that the operations of the cerebellar cortex in general and those of the climbing fiber system specifically are critical to real time operations performed in this structure. Furthermore the observations indicate that heterosynaptic actions of the climbing fiber system may be very important to cerebellar cortical function, not by generating long-term depression of synaptic responsiveness of Purkinje cells to parallel fiber inputs, but by producing short-term enhancement of these cells' responses to the mossy fiber-parallel fiber system. Interestingly, Hounsgaard's laboratory ${ }^{28}$ has shown that climbing fiber inputs can produce a comparable enhancement of synaptically-evoked depolarizations in Purkinje cells recorded in an in vitro preparation.

\section{CEREBELLAR PATIENT TRACING}
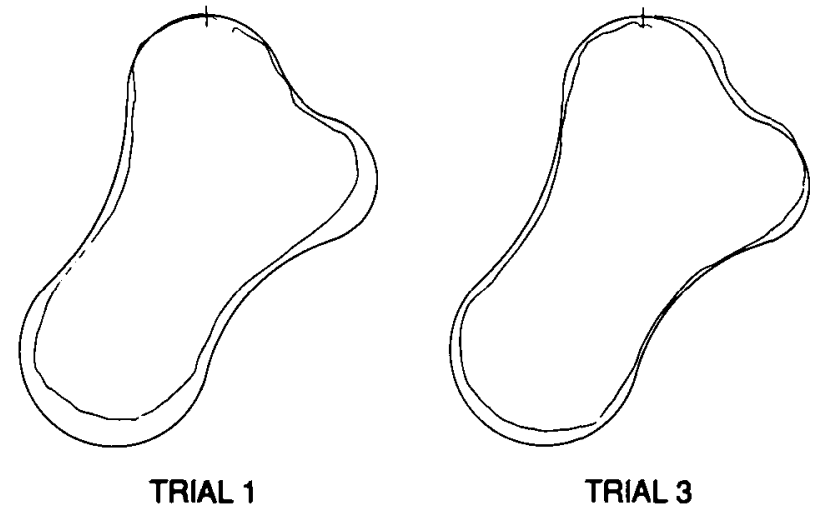

\section{NO OUTLINE $90^{\circ}$ ROTATION TO THE LEFT}

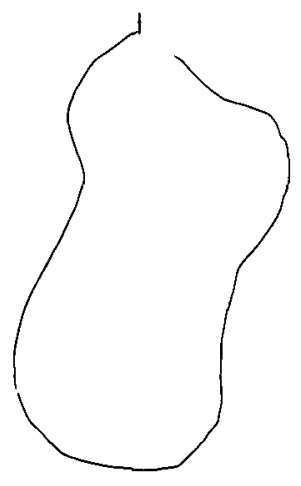

TRIAL 1

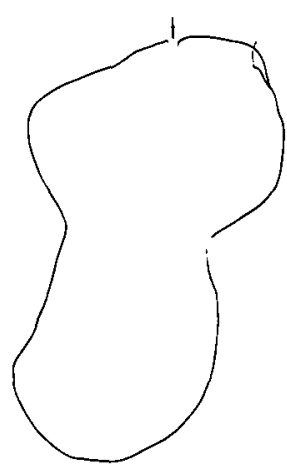

TRIAL 10
Figure 7 - Ability of a cerebellar patient to perform the same experiments as performed by the normal subject in Figure $6.1 n$ both the tracing task (upper drawings) and the drawing of the rotated figure (bottom drawings) the last trial shown is the one at which the subject reached maximal performance.

Because this enhanced responsiveness could be demonstrated for both excitatory as well as inhibitory responses of the Purkinje cells, Bloedel and Ebner ${ }^{29}$ formulated the gain change hypothesis as a descriptive framework for characterizing these interactions. According to this view the climbing fibers, when activated within a critical period prior to the arrival of other synaptic inputs to Purkinje cells, are capable of inducing an enhanced responsiveness or an increased gain of the neuron's response to its other synaptic inputs. As stated previously ${ }^{7}$ we further propose that this heterosynaptic effect of the climbing fiber system works together with its direct excitatory action on Purkinje cells in modulating the activity of cerebellar nuclear neurons.

In our view the dramatic enhancement of simple spike responsiveness illustrated in the findings presented above is much more likely to be relevant to the critical information processing performed by the cerebellar cortex than is long-term depression (See ${ }^{7,30}$ for further discussion). As indicated above, long-ter $m$ depression has never been observed in any condition 
employing natural stimulation. Secondly, it can only be produced by rates and durations of climbing fiber activation that are highly unlikely to occur for periods of several seconds in intact animals. In fact it appears that when low rates are employed to activate the climbing fiber system, the consistency of producing long-term depression is much less than when higher, sustained rates are used. Recently Crepel and his colleagues ${ }^{10}$ demonstrated a long-term depression in only $23 \%$ of neurons studied using a technique combining stimulation of the climbing fiber input at $\mathbf{I}$ $\mathrm{Hz}$ with the iontophoretic activation of Purkinje cells. Rather enhancement of responses was observed in many cells, a finding consistent with those in our laboratory reviewed above (Figure 1). Furthermore long-term depression usually is not a reversible experimental phenomenon. It is possible that the mechanisms underlying long-term depression may be more relevant to developmentally- or environmentally-induced trophic modifications of cerebellar circuitry than to a direct involvement in the plastic processes underlying motor learning.

The concept that the climbing fiber system is in part responsible for regulating the Purkinje cell's gain to its other synaptic inputs has been incorporated into a view of cerebellar cortical interactions designated the dynamic selection hypothesis. ${ }^{7.31}$ This hypothesis proposes a framework in which this interaction could be utilized in the cerebellar sagittal zone in a functionally meaningful way and more generally attempts to resolve one of the most enigmatic features of cerebellar organization. As emphasized by Voogd and colleagues, ${ }^{32-34}$ the concept of the sagittal zone describes two organizational components of the cerebellar cortex, the pattern of termination of the olivocerebellar fibers originating from specific locations within the contralateral inferior olive and the distribution of Purkinje cells terminating in restricted regions of the cerebellar nuclei. Since there is substantial overlap between the zonations describing each of these components of cerebellar cortical organization, the sagittal zone clearly comprises a unique input/output relationship between one of the afferent systems to the cerebellar cortex and the output neurons of this structure. Enigmatically, the afferent system responsible for carrying graded information about the periphery and activity in central pathways, namely the mossy fiber system, is not organized physiologically in the same way. The distribution of mossy fiber inputs from a specific body region has been described as a patchy mosaic by Welker and his colleagues. ${ }^{35}$ In general these inputs project to numerous small patches distributed to several regions of the cerebellar cortex. This organization provokes a critical question regarding the way in which the integration of multisensory information carried by the mossy fiber inputs is implemented in the context of the sagittal zones.

The dynamic selection hypothesis was proposed to provide a mechanism by which task-specific conditions could result in a localized, spatially-selective modulation of cerebellar efferent projections despite the wide distribution of mossy fibers likely to be activated in association with any motor behavior. This view proposes that the distribution of climbing fiber inputs activated under behavioral conditions is task specific, with the context of the behavior determining the patterns of converging inputs to discrete regions of the inferior olive. Consequently, even though the activated mossy fiber inputs may be widely distributed, the coincident activation of climbing fiber inputs to restricted regions of the sagittal zones could act to specify which populations of Purkinje cells may be most dramatically modulated. Given the organization of the corticonuclear projection, this interaction would further act to produce a modulation of specific populations of neurons in the cerebellar nuclei and their targets in the brainstem.

In summary the dynamic selection hypothesis ${ }^{7.31}$ proposes a mechanism by which the climbing fiber inputs can act to specify specific spatial distributions of Purkinje cells that will be most responsive and hence most highly modulated by combinations of mossy fiber inputs originating from many sources. This view requires a task-specific, behaviorally-dependent activation of specific populations of olivary neurons reflecting various patterns of converging inputs to the inferior olive. Although undoubtedly quite oversimplified, this proposal emphasizes a very critical conceptual point: the involvement of the olivocerebellar system in on-line, real time processing rather than in processes restricted to the establishment of memory traces in the cerebellum.

\section{The Cerebellum and Motor Learning}

The above data imply that the principal role of the cerebellum in motor control is not likely restricted to serving as a storage site for engrams established during motor learning. Our data, reviewed above, indicate that NMR conditioning can occur in decerebrate rabbits in the absence of the cerebellum. In addition, positive evidence was presented showing that temporary ablations of extracerebellar sites in the brainstem can produce selective reductions in the conditioned behavior. The recent multiple single unit recording data indicate that some cells in this region undergo changes in discharge properties related to the time course of the acquisition process rather than features of either the sensory stimulus or behavior of the NMR.

The possibility that the effects of cerebellar ablation are the consequence of the procedure on motor performance rather than motor learning is consistent with several studies in the literature, most notably those from John Harvey's laboratory. First, he and his colleagues showed that the selective effects of ablating regions of the cerebellar nuclei on the conditioned responses are principally the consequence of a performance deficit rather than a memory or learning deficit. ${ }^{36}$ This group also demonstrated that the microinjection of lidocaine into the critical regions of the interposed and/or dentate nuclei does not block the acquisition of the conditioned response despite the fact that the execution of the conditioned behavior is suppressed for the duration of the lidocaine block. ${ }^{37}$

Recently Nordholm et al. ${ }^{38}$ reported a study arguing that the effects of cerebellectomy reported by our laboratory ${ }^{2.3}$ reflected a set of observations characteristic of only the decerebrate preparation and that the reported responses may not even represent conditioned behavior. However there are several points regarding their study which indicate that their conclusions regarding our experiments may not be valid. First of all their study was performed in awake intact rabbits, whereas ours used decerebrate animals. Clearly some differences in temporal characteristics of the effective intertrial and interstimulus intervals would be expected, since structures above the midbrain are considered to have at least a modulatory role in NMR conditioning and behavior. ${ }^{39-43}$ They also argued that our criteria for CR threshold was low enough to result in the counting of a substantial number of spontaneous eye blinks as conditioned responses. 
However in our preparations spontaneous eye blinks were essentially nonexistent and our baselines were extremely stable, since the head of the animals and the apparatus were fixed and stabilized rather than being attached by an elastic band, as in the studies of Nordholm et al. ${ }^{38}$ Furthermore we demonstrated that the responses we scored as CRs were tightly coupled to the onset of the US when the interstimulus interval was changed, a feature which would not be expected for either unconditioned or conditioned alpha responses. Based on these problems with the arguments of Nordholm et al. ${ }^{38}$ and the clear demonstration of conditioning in our decerebellate, decerebrate rabbits, we conclude that the previous observations of Thompson and colleagues reflect the consequence of the cerebellar lesion on the excitability of brainstem reflexes rather than the loss of the site required for the storage of the memory trace.

The likelihood that cerebellar lesions result primarily in alterations of motor performance rather than the loss of a critical storage site for motor learning also is consistent with the human studies presented above (Figures 6 and 7). This patient could clearly improve his performance, an indication that motor learning did occur despite the extensive cerebellar pathology. The principal deficit related to an inability to properly integrate available sensory cues, a feature that will be discussed more extensively below. It should be emphasized that cerebellar patients assessed in other laboratories ${ }^{16}$ also were capable of improving motor performance with practice.

Based on an overview of all the available data, including several findings on the substrates responsible for vestibulo-ocular reflex adaptation which are not reviewed here, we find it difficult to support the notion that the cerebellum serves as a necessary and sufficient storage site for the plastic processes underlying most types of motor learning. Rather the data imply an involvement of this structure in the acquisition process and in the on-line interactions responsible for regulating motor coordination. This view does not rule out the possibility that plastic changes occur in the cerebellum. However it does propose a primary cerebellar function focused on sensorimotor integration rather than memory storage.

\section{Cerebellar Function}

In our view cerebellar function is best characterized as a singular operation that is critical for the performance of novel coordinated movements. ${ }^{7}$ As indicated in the first section of this overview, this function probably involves the combined action of mossy and climbing fiber projections in a manner that imple- ments the sagittal zone and its precise connections with extracerebellar structures.

The precise algorithms representing the operations performed by the cerebellar cortex and nuclei are currently unknown. However substantial insights are available in the literature regarding the most critical aspects of the information processing occurring in this structure. Based on a model of sensory-motor processing, Pellionisz and Llinas ${ }^{44}$ and subsequently Pellionisz ${ }^{45}$ argued that the cerebellum participates in coordinate transformations required for utilizing sensory inputs in a manner that optimizes the performance of a movement. A review of the pertinent literature ${ }^{7}$ suggests that the most critical of these transformations involves the integration of visually-mediated information about external target space with information characterizing proprioceptive and exteroceptive cues, the internal representation of the target space, body scheme, ${ }^{46}$ and activity in motor pathways. Specific studies in both humans and animals suggest that this type of integration cannot occur in the presence of large cerebellar lesions. ${ }^{7,47-49}$

As presented in Figure 7, the availability of a patient with a selective, MRI-documented lesion of one cerebellar hemisphere following a severe cerebellar stroke made it possible to examine certain predictions suggested by this view. It was assumed that if the cerebellum was critical for integrating teleceptive information with inputs reflecting characteristics of ongoing motor behavior and/or the target of the executed movement, a patient with a substantial lesion of this structure should be unable to effectively generate movements that require the intrinsic calculations and/or axis rotations used in these drawing tasks. This patient was clearly unable to perform these movements, even with repeated practice. It should be emphasized that he was able to improve his performance on standard tracing tasks even though the movements remained ataxic. Although very preliminary, these data support the implications of previous studies ${ }^{47-49}$ indicating that tasks requiring relatively complex visuomotor processing are highly dependent on the cerebellum for their performance.

Figure 8 integrates this view of cerebellar integration with the notion that this structure plays an important role in motor learning without serving as a required storage site for the plastic changes involved in this process. This scheme emphasizes that the output of the cerebellum projects to regions that are critical for both task performance as well as task acquisition. It further implies that the capacity to perform the movement in a coordinated manner is a prerequisite for it to be learned, at least if

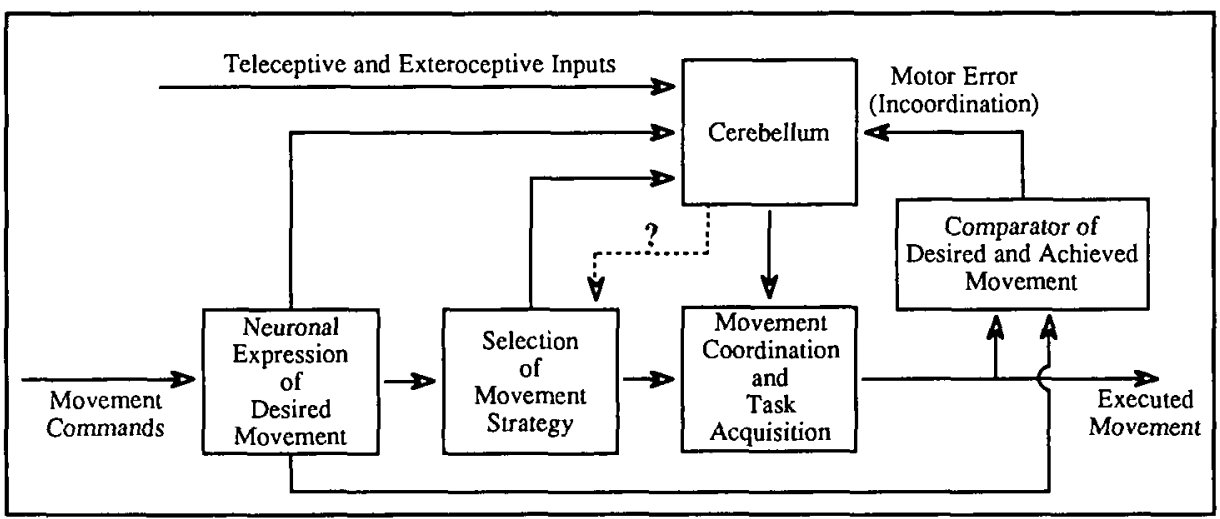

Figure 8 - Diagram illustrating interactions by which the cerebellum could play a critical role in task acquisition and the synthesis of movement strategies without serving as the storage site for the memory traces established during motor learning. 
learning is assessed by the improvement in motor skill demonstrated as the movement is executed over successive trials.

In conclusion, the above overview emphasizes two critical concepts. First, cerebellar function is best described in terms of a singular operation performed by the circuitry of the cerebellar cortex and nuclei. This operation implements the sagittal zone and the related topographical features of cerebellar output projections in modifying the information processing in extracerebellar nuclei critical for motor execution. Second, the most critical type of integration performed by the cerebellum involves the synthesis of visuomotor information with multiple peripheral and central inputs activated as the movement is prepared and executed.

\section{ACKNOWLEDGEMENTS}

The authors wish to thank Mr. Joel McAlduff and Ms. Michelle Webster for their technical help during the course of the experiments and Ms. Jan Carey for her assistance in the preparation of the manuscript. The research was performed with funds from NIH grant (NS 21958) and training grant funds from NIH grant (NS 07309).

\section{REFERENCES}

1. Ito M. The cerebellar control of the vestibulo-ocular reflex around the flocculus hypothesis. Ann Rev Neurosci 1982; 5: 275-296.

2. Ito $M$. The role of the cerebellum during motor learning in the vestibular reflex: different mechanisms and different species. Trends Neurosci 1982; 5: 416

3. McCormick DA, Thompson RF. Neuronal responses of the rabbit cerebellum during acquisition and performance of a classically conditioned nictitating membrane-eyelid response. J Neurosci 1984; 4: 2811-2822.

4. Thompson RF. The neurobiology of learning and memory. Science 1986; 223: $941-947$.

5. Yeo $\mathrm{CH}$, Hardiman MJ, Glickstein M. Classical conditioning of the nictitating membrane response of the rabbit. II. Lesions of the cerebellar cortex. Exp Brain Res 1985; 60: 99-113.

6. Yeo $\mathrm{CH}$, Hardiman MJ, Glickstein M. Classical conditioning of the nictitating membrane response of the rabbit. I. Lesions of the cerebellar nuclei. Exp Brain Res 1985; 60: 87-98.

7. Bloedel JR. Functional heterogeneity with structural homogeneity: how does the cerebellum operate? Behav Brain Sci 1992: in press.

8. Wilson VJ, Yamagata $\mathrm{Y}$, Yates BJ, et al. Response of vestibular neurons to head rotations in vertical planes. III. Response of vestibulocollic neurons to vestibular and neck stimulation. J Neurophysiol 1990; 64: 1695-1703.

9. McCormick DA, Thompson RF. Cerebellum: essential involvement in the classically conditioned eyelid response. Science 1984; 223: 296-299.

10. Crepel F, Jaillard D. Pairing of pre- and post-synaptic activities in cerebellar Purkinje cells induces long-term changes in synaptic efficacy in vitro. J Physiol 1991; 432: 123-141.

11. Sakurai M. Synaptic modification of parallel fiber - Purkinje cell transmission in in vitro guinea pig cerebellar slices. J Physiol 1987; 394: 463-480.

12. Knowlton BJ, Lavond DG, Thompson RF. The effect of lesions of cerebellar cortex on retention of the classically conditioned eyeblink response when stimulation of the lateral reticular nucleus is used as the conditioned stimulus. Behav Neural Biol 1988; 49: 293-301.

13. Lavond DG, Knowlton BJ, Steinmetz JE, et al. Classical conditioning of the rabbit eyelid response with mossy fiber stimulation as a CS. II. Lateral reticular nucleus stimulation. Behav Neurosci 1987; 101: 676-682.

14. Mauk MD, Steinmetz JE, Thompson RF. Classical conditioning using stimulation of the inferior olive as the unconditioned stimulus. Proc Natl Acad Sci USA 1986; 83: 5349-5353.

15. Steinmetz JE, Lavond DG, Thompson RF. Classical conditioning of the rabbit eyelid response with mossy fiber stimulation as the conditioned stimulus. Bull Psychon 1985; 23: 245-248.
16. Sanes JN, Dimitrov B, Hallett M. Motor learning in patients with cerebellar dysfunction. Brain 1990; 113: 103-120.

17. Ebner TJ, Bloedel JR. Role of climbing fiber afferent input in determining the responsiveness of Purkinje cells to mossy fiber inputs. J Neurophysiol 1981; 45: 962-971.

18. Schwartz AB, Ebner TJ, Bloedel JR. Comparison of responses in dentate and interposed nuclei to perturbations of the locomotor cycle. Exp Brain Res 1987; 67: 323-338.

19. Lou J-S, Bloedel JR. The responses of simultaneously recorded Purkinje cells to perturbations of the step cycle in the walking ferret: a study using a new analytical method - the real time postsynaptic response (RTPR). Brain Res 1986; 365: 340-344.

20. Ebner TJ, Yu Q-X, Bloedel JR. Increase in Purkinje cell gain associated with naturally activated climbing fiber inputs. J Neurophysiol 1983; 50: 205-219.

21. Ebner TJ, Bloedel JR. Climbing fiber action on the responsiveness of Purkinje cells to parallel fiber inputs. Brain Res 1984; 309: 182-186.

22. Bloedel JR, Ebner TJ, Yu Q-X. Increased responsiveness of Purkinje cells associated with climbing fiber inputs to neighboring neurons. J Neurophysiol 1983; 50: 220-239.

23. Kelly TM, Zuo C-C, Bloedel JR. Classical conditioning of the eyeblink reflex in the decerebrate-decerebellate rabbit. Behav Brain Res 1990; 38: 7-18.

24. Bracha V, Wu J-Z, Cartwright $S$, et al. Selective involvement of the spinal trigeminal nucleus in the conditioned nictitating membrane reflex of the rabbit. Brain Res 1991; 556: 317-320.

25. Miller S, Van der Burg J, Van der Meche FGA. Locomotion in the cat: basic programmers of movement. Brain Res 1975; 91: 239-253.

26. Desmond JE, Moore JW. A supratrigeminal region implicated in the classically conditioned nictitating membrane response. Brain Res Bull 1983; 10: 765-773.

27. Bracha V, Cartwright $M$, Bloedel JR. Multiple single unit recording during the conditioning of the nictitating membrane reflex in the rabbit. Proc Third IBRO World Congress 1991: 173 (Abstract).

28. Chan CY, Hounsgaard J, Midtgaard J. Excitatory synaptic responses in turtle cerebellar Purkinje cells. J Physiol 1989; 409: 143-156.

29. Bloedel JR, Ebner TJ. Climbing fiber function: regulation of Purkinje cell responsiveness. In: Bloedel JR, Dichgans J, Precht W, eds. Cerebellar Functions. Berlin, Heidelberg, New York: Springer-Verlag, 1985: 247-259.

30. Bloedel JR, Zuo C-C. The heterosynaptic action of climbing fibers in the cerebellar cortex. Exp Brain Res 17 1989; 246-264.

31. Bloedel JR, Kelly TM. The Dynamic Selection Hypothesis: A Proposed Function for Cerebellar Sagittal Zones. In: Llinas R, Soleto C, eds. The Cerebellum Revisited. New York: SpringerVerlag, 1992: 267-282.

32. Groenewegen HJ, Voogd J. The parasagittal zonation within the olivocerebellar projection. I. Climbing fiber distribution in the vermis of cat cerebellum. J Comp Neurol 1977; 174: 417-488.

33. Groenewegen HJ, Voogd J, Freedman SL. The parasagittal zonation within the olivocerebellar projection. II. Climbing fiber distribution in the intermediate and hemispheric parts of cat cerebellum. J Comp Neurol 1979; 183: 551-602.

34. Voogd J, Bigare F. Topographical distribution of olivary and cortico nuclear fibers in the cerebellum: a review. $I n$ : Courville J, de Montigny C, Lamarre Y, eds. The Inferior Olivary Nucleus: Anatomy and Physiology. New York: Raven Press, 1980: 207-234.

35. Shambes GM, Gibson JM, Welker W. Fractured somatotopy in granule cell tactile areas of rat cerebellar hemispheres revealed by micromapping. Brain Behav Evol 1978; 15: 94-140.

36. Welsh JP, Harvey JA. Cerebellar lesions and the nictitating membrane reflex: performance deficits of the conditioned and unconditioned response. J Neurosci 1989; 9: 299-311.

37. Welsh JP, Harvey JA. Pavlovian conditioning in the rabbit during inactivation of the interpositus nucleus. J Physiol 1991; 444: 459-480.

38. Nordholm AF, Lavond DG, Thompson RF. Are eyeblink responses to tone in the decerebrate, decerebellate rabbit conditioned responses? Behav Brain Res 1991; 44: 27-34.

39. Akase E, Alkon DL, Disterhoft JF. Hippocampal lesions impair memory of short-delay conditioned eye-blink in rabbit. Behav Neurosci 1989; 103: 935-943. 
40. Megirian D, Bures $\mathbf{J}$. Unilateral cortical spreading depression and conditioned eyeblink responses in the rabbit. Exp Neurol 1970; 27: $34-45$.

41. Moyer Jr, J.R., Deyo RA, Disterhoft JF. Hippocampectomy disrupts trace eye-blink conditioning in rabbits. Behav Neurosci 1990; 104: 243-252.

42. Oakley DA, Russell IS. Subcortical nature of Pavlovian differentiation in the rabbit. Physiol Behav 1976; 17: 947-954.

43. Salafia WR, Nicolas LC, Ramirez JJ. Retardation of rabbit nictitating membrane conditioning by subseizure electrical stimulation of hippocampus. Physiol \& Behav 1979; 22: 451-455.

44. Pellionisz A, Llinas R. Tensor network theory of the metaorganization of functional geometries in the central nervous system. Neuroscience 1985; 16: 245-273.

45. Pellionisz AJ. Tensorial brain theory in cerebellar modelling. In: Bloedel JR, Dichgans J, Precht W, eds. Cerebellar Functions. Berlin, Heidelberg, New York, Tokyo: Springer-Verlag, 1985: 201-229.
46. Lestienne F, Gurfinkel VS. Posture as an organizational structure based on a dual process: a formal basis to interpret changes of posture in weightlessness. In: Pompeiano O, Allum J, eds. Vestibulospinal control of posture and locomotion. Prog Brain Res 1988; 76: 307-313.

47. Beppu H, Nagaoka M, Tanaka R. Analysis of cerebellar motor disorders by visually-guided elbow tracking movement. Brain 1987; 110:1-18.

48. Beppu H, Suda M, Tanaka R. Analysis of cerebellar motor disorders by visually guided elbow tracking movement. Brain 1984; 107: 787-809.

49. Vercher J-L, Gauthier GM. Cerebellar involvement in the coordination control of the oculo-manual tracking system: effects of cerebellar dentate nucleus lesion. Exp Brain Res 1988; 73: 155-166. 